\title{
Aproximaciones socioantropológicas al estudio de los grupos de Whatsapp y Facebook
}

\section{Abordagens sócio-antropológicas para o estudo dos grupos de Whatsapp e Facebook}

Mercedes Durá-Lizán ${ }^{1}$

Antonio Miguel Nogués-Pedregal ${ }^{2}$

Resumen: Este artículo presenta un análisis comparado de la bibliografía sobre los intercambios comunicativos que se producen en los grupos de carácter público en WhatsApp y Facebook. La metodología aplicada ha sido la búsqueda sistemática de artículos científicos en Web of Science y Scopus, y de los textos disponibles en castellano y portugués en Google Académico, lo que nos ha permitido seleccionar un corpus final de 56 artículos científicos, que hemos organizado $y$ sintetizado en función de los enfoques teóricos, temas y métodos. Concluimos que las diferencias encontradas en las metodologías de investigación responden a que nos encontramos ante escenarios tecnológicos en los que se producen interacciones que tiene distinto significado para los actores, más próximo a la conversación en los grupos de WhatsApp y al relato en los de Facebook.

Palabras clave: WhatsApp; Facebook; grupos digitales; etnografía digital; affordance

Resumo: Este artigo apresenta uma análise comparativa da bibliografia sobre as trocas comunicativas que acontecem nos grupos públicos no WhatsApp e no Facebook. A metodologia aplicada foi a pesquisa sistemática de artigos científicos

1 Universidad Miguel Hernández (UMH). Elche, Alicante, Espanha. http://orcid.org/0000-0002-8128-437X E-mail: mdura@umh.es

2 Universidad Miguel Hernández (UMH). Elche, Alicante, Espanha. http://orcid.org/0000-0002-8352-4488 E-mail: amnogues@umh.es 
na Web of Science e Scopus, e dos textos disponíveis em espanhol e português no Google Scholar, o que nos permitiu acessar a um corpus final de 56 artigos científicos, que temos organizado e sintetizado em função das abordagens teóricas, temas e métodos. Concluímos que as diferenças encontradas nas metodologias de pesquisa respondem ao fato de estarmos diante de cenários tecnológicos nos quais acontecem interações que têm significados diferentes para os atores, mais próximos da conversa nos grupos de WhatsApp e da narração no Facebook.

Palavras-chave: WhatsApp; Facebook; grupos digitais; etnografia digital; affordance 


\section{Introducción}

La proliferación de grupos digitales circunscritos a un territorio o una temática concreta demuestra que estos se han convertido en escenarios privilegiados para la comunicación pública. Los colectivos sociales crean estos grupos usando, sobre todo, la red social Facebook o bien la aplicación de mensajería instantánea WhatsApp, decantándose por una u otra opción en función de la fortaleza de los vínculos, el encuadramiento temático o las características del contexto que rodea a sus miembros (DURÁ-LIZÁN; TRAVÉ-MOLERO; NOGUÉS-PEDREGAL, 2020).

A pesar de su relevancia social, son escasas las investigaciones en torno a los grupos digitales de redes sociales, ya sea como objeto de estudio o como campo de investigación etnográfico. La necesidad de adaptar la metodología y técnicas a los nuevos entornos tecnológicos (ÁLVAREZ GANDOLFI, 2016a; ARDÉVOL; GÓMEZ-CRUZ, 2013), las incertidumbres éticas y legales (BARBOSA; MILAN, 2019; ESTALELLA; ARDĖVOL, 2007) e incluso cuestiones relacionadas con la autenticidad de los datos (HINE, 2015), pueden ser un freno a la investigación.

Esta revisión bibliográfica busca organizar, resumir y contrastar las perspectivas, temas y metodologías predominantes desde las que se ha abordado la incipiente investigación sobre la naturaleza de los intercambios comunicativos que se producen en los grupos de WhatsApp y Facebook.

Hemos dado prioridad a las perspectivas socioantropológicas, que aportan a la investigación de la comunicación el interés por las redes de socialización que articula y la comprensión de los sistemas comunicativos como procesos intersubjetivos en los que se producen significados sociales. Además, estos enfoques suelen asumir, de forma más o menos manifiesta, las prácticas comunicativas mediatizadas digitalmente como resultado del encuentro entre las potencialidades de la tecnología y las lecturas que hacen los usuarios de ella (DAVIS; CHOUINARD, 2016).

Priorizamos, igualmente, las aproximaciones etnográficas -o metodologías afines como el análisis de discurso-, que permiten acceder a las formas de interacción y a los significados profundos que se generan 
en los grupos creados en las plataformas digitales, y que ponen el foco en el contexto social como fuente fundamental de sentido. Dejamos a un lado las áreas aplicadas como el marketing, la salud o la educación.

Los textos han sido seleccionados, en primera instancia, a partir de las bases de datos de reconocida calidad científica Web of Science y Scopus. A continuación, hemos realizado una búsqueda intensiva de los artículos disponibles en castellano y portugués en Google Académico, ya que la producción en estos idiomas sobre el tema es reducida y la herramienta de Google permite acceder a un volumen mayor de documentos. En total, hemos analizado un corpus de 56 artículos científicos escritos en inglés, español y portugués. De ellos 30 estudian intercambios comunicativos en grupos de Facebook, 24 en grupos de WhatsApp y dos lo hacen en grupos creados en ambas plataformas. En cada uno de los textos hemos identificado los principales enfoques, temáticas, así como las metodologías y técnicas aplicadas.

A partir de este trabajo, hemos categorizado los textos en dos grandes bloques: (1) los artículos que estudian el flujo de conversación que se genera en WhatsApp y (2) los textos que estudian los relatos que se producen en Facebook.

\section{El estudio de las interacciones conversacionales en los grupos de WhatsApp}

El acceso a los grupos creados en WhatsApp, generalmente vinculados a comunidades cerradas, es especialmente difícil y controvertido para el investigador y puede ser la causa de que este objeto de estudio aún sea marginal. De hecho, es frecuente que las investigaciones en torno a la aplicación se realicen en contextos accesibles y relacionados con los propios ambientes universitarios. En este ambiente, se han investigado mediante la administración de cuestionarios los procesos a través de los cuales los jóvenes universitarios hacen suya esta tecnología y los sentidos que adquiere en su socialización (AHAD; LIM, 2014), la relevancia que el sentimiento de apego tienen en la motivación para participar en 
grupos de WhatsApp formados por familiares (KURNIASIH; RIYADHSYAH, 2018) o cómo la discusión de los jóvenes en torno a noticias de actualidad en grupos de WhatsApp estimula en ellos el interés por la política (VERMEER et al., 2020).

Virginia Fuentes, Marta García y María Aranda (2017) han estudiado las dinámicas comunicativas en los grupos de WhatsApp vinculados a materias universitarias, usando como técnica los grupos de discusión. Destacan el dinamismo que introduce WhatsApp en la comunicación grupal y argumentan que a través de esta aplicación se modifican "las maneras de proceder y relacionarse, los elementos considerados necesarios para la inclusión/exclusión de las personas, los códigos empleados para la comunicación y el propio sentimiento de identidad y/o pertenencia" (2017, p. 167). Sin embargo, advertimos que esta investigación tiene una naturaleza sincrónica por lo que las posibilidades de dar cuenta de dicha modificación son limitadas.

Más allá de los entornos académicos, Kenton P. O'Hara y sus compañeros (2014) investigan los grupos de WhatsApp relacionados con la residencia mediante grupos de discusión, y detectan que los encuentros cotidianos y el 'hacer juntos' dan sentido y sustentan la existencia de estos grupos. Este proceso se ubica dentro de lo que Rich Ling y Chih-hui Lai (2016) denominan 'micro-coordinación 2.0', concepto que designa la modalidad de coordinación que se produce con la incorporación de la conectividad digital a los teléfonos móviles. Estos autores también destacan que los chats grupales están formados normalmente por personas que comparten un marco contextual común. En esta línea se ha investigado la coordinación de los trabajadores rurales de Kenya e India en los grupos de WhatsApp creados por empresas distribuidoras (MCGREGOR et al., 2019). Estos investigadores desarrollan un análisis etnometodológico de las conversaciones que se producen en grupos de discusión, en los que los propios participantes explican el sentido de sus interacciones en el entorno digital.

Las investigaciones antes citadas sobre grupos de WhatsApp todavía se basan en lo que los miembros dicen que hacen, en encuestas, 
entrevistas o grupos de discusión, pero no investigan las prácticas concretas, es decir, los momentos de producción de los mensajes y las interacciones que tienen lugar en estos escenarios. Cristina Vela Delfa y Lucía Cantamutto (2016) defienden el uso de la observación participante de los intercambios que se producen en esta aplicación para conocer sus características estructurales y organizativas, así como el modo en que influyen en los sistemas de relaciones sociales en los que se insertan.

Algunas etnografías usan como campo indistintamente WhatsApp y lugares presenciales de interacción. Desde este enfoque metodológico, Robson da Silva Braga (2016) ha indagado sobre el modo en que los nordestinos afincados en Rio de Janeiro usan WhatsApp para coordinar sus quedadas en la ciudad. Según el autor, la creación de lugares de encuentro promueve vínculos que les permiten fortalecer su identidad regional. Con esta misma dimensión territorial urbana y un trabajo de campo en línea y fuera de línea, se ha estudiado la coordinación y participación en materia de seguridad en grupos públicos de WhatsApp en sectores urbanos de los Países Bajos (MOLS; PRIDMORE, 2019) y de Córdoba en Argentina (TORRES, 2017).

Esta función de coordinación es clave para colectivos cohesionados y organizados en torno a causas o proyectos. Así, Nicolás Aguilar-Forero (2017) estudia las tácticas de apropiación tecnológica de H.I.J.O.S. Bogotá, organización que reivindica la reparación a las víctimas del conflicto en Colombia, combinando técnicas propias del trabajo de campo con el análisis de los discursos digitales. El autor describe los diferentes espacios dedicados a la comunicación con un enfoque multilocal, y destaca que

las tecnologías digitales y los mundos virtuales tienen enormes potencialidades no solo para la circulación de contenidos y la coordinación de acciones colectivas, sino también para propiciar el encuentro on/ offline, promover el intercambio de información, consolidar los vínculos humanos y, en especial, canalizar las energías de la confrontación (AGUILAR-FORERO, 2017, p. 144). 
Por su parte, Janine de Kássia Bargas y Rousiley Celi Maia (2017) han investigado el proceso de aprendizaje colectivo en el que participan jóvenes de comunidades tradicionales de Brasil que forman parte de un grupo de WhatsApp sobre el Processo Seletivo Especial de la Universidad Federal de Pará. Usando como técnicas la observación participante y la sistematización de las conversaciones, detectan que el grupo tiene las siguientes funciones: 1) proveer información, aumentando la confianza que existía previamente entre los miembros; 2) la expresión del posicionamiento, que permite ajustar entre sí las diferentes opiniones; y 3 ) la expresión de sugerencias, a fin de facilitar la solución de problemas del grupo.

Las posibilidades ofrecidas por la aplicación WhatsApp para observar y analizar las conversaciones han despertado el interés por la naturaleza de estas, como vemos también en la investigación etnográfica, desarrollada mediante entrevistas en profundidad y observación participante en línea, de las funciones de las interacciones -intercambio, competencia, cooperación, conflicto y coerción- que se producen en un grupo de profesores de Indonesia (KURNIASIH; RIYADHSYAH, 2018).

Algunas características como la rapidez del intercambio de mensajes, el carácter privado e informal, el escaso rastro que dejan las interacciones, así como la posibilidad de difundir mensajes en red, convierten a la aplicación WhatsApp en una herramienta fundamental para difundir con éxito peticiones de justicia en la India, mediante las cuales la población civil solicita el apoyo local y la autorización administrativa para dar caza a grandes felinos que han atacado a humanos (MATHUR, 2019). La aplicación de mensajería agiliza el proceso, al permitir que se produzca sin que trascienda a la opinión pública, ni adquiera estatus oficial.

Con un enfoque etnometodológico apoyado en la observación en línea, Marco Túlio de Souza (2018) describe las prácticas de coordinación relacionadas con la oración en un grupo de WhatsApp, y concluye que el uso de esta aplicación por parte de comunidades religiosas transforma la vivencia del tiempo discontinuo y el espacio exclusivo característicos de lo sagrado, aproximándolo a la vida ordinaria. 
El interés en el análisis de contenidos ha estado estimulado en Brasil por el repentino éxito de WhatsApp en la campaña presidencial de Jail Bolsonaro, que reveló que pese a la aparente simplicidad de la aplicación de mensajería es posible movilizar contenidos de carácter ideológico a través de ella (CHAGAS; MODESTO; MAGALHÃES, 2019; EVANGELISTA; BRUNO, 2019; RESENDE et al., 2018).

Debido a la potencialidad de WhatsApp para fijar por escrito conversaciones informales, encontramos análisis conversacionales que desde el campo de la sociolingüística detectan las estrategias empleadas para plasmar por escrito aspectos característicos de la comunicación oral, como los emojis, el uso de interjecciones, la repetición de letras y signos, el uso de mayúsculas, marcadores conversaciones no semánticos o las partículas de risa (PÉREZ-SABATER, 2015). A esta escritura fonética que resulta de la trasposición del lenguaje oral al escrito, se conoce también como 'internetes' (DOS SANTOS; SILVA, 2018). En ocasiones estos análisis se centran en algunos aspectos concretos de este fenómeno, como las 'reacciones confirmatorias' (PADILLA HERRADA, 2017), las piezas humorísticas (DONCEL, 2016), o las partículas de risa y los emojis (KÖNIG, 2019).

\section{El análisis de discurso como método etnográfico en los grupos de Facebook}

Las investigaciones en grupos digitales de Facebook se abordan con mayor frecuencia desde el análisis de discurso, con el objetivo de conocer de qué modo se desarrollan los procesos de producción ideológica e identitaria en estos entornos. En este sentido, una parte de la literatura científica se ha interesado por la producción de identidades alternativas construidas en torno a nuevas producciones culturales, como los fanes de series de televisión (TUR-VIÑES; FERRÁNDIZ, 2014), seguidores de la cultura otaku (ÁLVAREZ GANDOLFI, 2016b) o los otherkin, que se identifican con una naturaleza no humana (PROCTOR, 2018).

Varios investigadores han aplicado el análisis de discurso para conocer los procesos de producción de ideologías políticas en los grupos 
creados en Facebook (ACOSTA, 2012; MARICHAL, 2013). Especial atención han despertado los grupos creados por minorías para fortalecer la identificación colectiva en torno a un idea política, como es el caso de aquellos que promueven estilos de vida energéticamente sostenibles (REVILLA, 2020), los grupos de jóvenes activistas salvadoreños (HARLOW, 2016), la reivindicación de los docentes filipinos contra el denostado estatus profesional del maestro contemplado en la reforma educativa (ARZADON, 2016), los grupos con una orientación feminista en España (SÁDABA; BARRANQUERO, 2019), o aquellos producidos por las mujeres sudanesas que proponían una nueva narrativa sobre las políticas del cuerpo (ALI, 2019).

También se ha prestado interés a los contenidos que se producen en los grupos de Facebook creados en torno a ideologías reaccionarias. Así, Daniela Meneses (2019) realiza una etnografía y un análisis de discurso del grupo Con Mis Hijos No Te Metas PERÚ-Oficial, que se opone a la reforma educativa del gobierno peruano y a la ideología de género. Del mismo modo, se ha observado que la interacción en espacios abiertos contribuye a normalizar los discursos racistas en un grupo de Facebook que se posiciona en contra de la migración y los refugiados (EKMAN, 2019), así como la construcción del discurso de odio en las campañas presidenciales de Colombia (TABARES HIGUITA, 2018) o la creación de visiones reduccionistas de la historia nacional mexicana (ITURRALDE CHAPARRO, 2019). También se han explorado -en este caso mediante una etnografía clásica basada en entrevistas- la imagen negativa sobre la juventud que se construye en un grupo orientado a la discusión comunitaria en una ciudad australiana (CLIFFORD; SMITH; CHRISTIE, 2020).

Un campo de observación interesante son los grupos formados por colectivos que se caracterizan por estar viviendo un proceso especialmente activo de construcción identitaria. Es el caso del estudio de la performatividad de género en personas transfemeninas, mediante el análisis de los mecanismos de auto-representación de las participantes discursos y fotografías-, en un grupo de Facebook creado expresamente 
con este fin (GUTIÉRREZ MARTÍNEZ, 2016). La investigadora detecta que el sistema de relaciones que desarrollan en la plataforma coincide y refuerza su red de relaciones presenciales habituales.

Por medio de una etnografía visual, se han examinado también las prácticas performativas en grupos de Facebook que permiten a los jóvenes chilenos proyectar una imagen positiva ante otros participantes coetáneos (RIQUELME; SOLÍS; GALLEGOS, 2018), o la correlación entre la forma en que los jóvenes cubanos usan Facebook y su propia visión de la vida (MARRERO SANTANA; JIMÉNEZ HERNÁNDEZ; RODRÍGUEZ BRITO, 2017).

Hemos encontrado un número significativo de investigaciones que abordan la producción identitaria en relación al territorio. En este sentido, los grupos creados por migrantes son un campo especialmente fecundo para observar los procesos de manutención de la identidad territorial simbólica, a pesar de la distancia física. Así pues, se ha investigado el uso que mexicanos asentados en Barcelona hacen de esta herramienta con el fin de contactarse y mantener su identidad mexicana (RAMÍREZ PLASCENCIA, 2020), las estrategias de compensación en términos de representación de la minoría Rumana en Turquía (GÜZEL KORVER; KESKIN, 2019) y las transformaciones que se producen en la identificación de los migrantes italianos asentados en Toronto (GIUS, 2019). Estas aproximaciones suelen compaginar la etnografía en línea y fuera de línea.

En otras ocasiones, los participantes de los grupos generan un relato territorial opuesto al que trasmiten los medios de comunicación de masas. Así, los habitantes de la región del volcán Merapi (Indonesia) generan en el grupo merapi.info un relato que realza la tradicional forma de vida armónica con la naturaleza y la belleza de lugar, frente a la imagen de peligrosidad dominante, como revela el Análisis Crítico de Discurso aplicado a los textos del grupo (NAZARUDDIN, 2020).

Otras veces, los miembros actualizan lugares que pertenecen al pasado con los que conservan un vínculo sentimental, como muestra la investigación desarrollada por María Lorena Capogrossi, Mariana 
Loreta Magallanes y Florencia Soraire (2015) sobre las vivencias en el Campamento Vespucio de Yacimientos Petrolíferos Fiscales, usando el grupo de Facebook de sus antiguos trabajadores como fuente. Las autoras consideran que

pueden señalarse los arraigos territoriales que construyen los usuarios en Facebook a partir de lo que allí cuentan. El territorio expuesto en espacios virtuales y visto como propulsor de la idea de comunidad, puede que nos esté diciendo algo sobre aquel núcleo de personas que estuvo arraigado en un espacio físico particular y que se ha reencontrado ahora en un espacio virtual o, en otras palabras, que se ha vuelto a agrupar en una comunidad, esta vez ritual (CAPOGROSSI; MAGALLANES; SORAIRE, 2015, p. 52).

En esta línea, se han examinado las reacciones a la perdida de patrimonio en la ciudad de Perth, en Australia Occidental, en el grupo de Facebook Beautiful buildings and cool places Perth has lost (GREGORY, 2015). Según la autora, en este espacio se genera una comunidad emocional que produce capital social, con fuerza suficiente para impulsar acciones concretas.

Otros estudios se han centrado en los grupos de Facebook ligados a la reivindicación de la autodeterminación regional. En esta línea, Koldo Díaz (2015) analiza la producción de la identidad territorial vasca en el grupo de Facebook Euskalduna naiz profileko argazkian mendian ez agertu arren -Soy vasco aunque en la foto de perfil no aparezca en el monte-. Utiliza este grupo como fuente y como campo para conocer los mecanismos que los miembros utilizan para apropiarse en internet de un territorio del que no pueden disponer sobre el espacio geográfico, al encontrarse dentro de las fronteras española y francesa.

En esta misma línea se ha investigado, mediante una etnografía estrictamente virtual, el modo en que los turco-chipriotas aumentan su autoestima nacional al destacar en sus grupos de Facebook sus características distintivas frente a la Turquía continental (AKÇALI, 2019), y las prácticas de resistencia lingüística en grupos creados para preservar el 
turco otomano ante la estrategia del gobierno orientada a promover su extinción (YAZAN, 2015).

En otras ocasiones, el uso de las herramientas digitales para construir una identidad territorial no proviene de un sentimiento nacionalista o regionalista, sino que ha sido inducido por promotores inmobiliarios para generar un sensación de vecindad entre los nuevos habitantes de un área de reciente urbanización (MOSCONI et al., 2017). En este sentido, algunas investigaciones valoran la aportación de los grupos a la producción de valor en los territorios a los que se vinculan (FERRAUDI CURTO, 2018; GARCÍA HUERTAS; GARRIDO PINTADO; BOTAS LEAL, 2018).

Algunos autores han investigado las prácticas que se producen en grupos creados en ambas plataformas. Este es el caso del análisis realizado mediante grupos de discusión por Joëlle Swart y sus colegas (2019) sobre las repercusiones del intercambio de noticias en grupos creados por comunidades que comparten una misma ubicación geográfica. Concluyen que las estructuras relacionales de los miembros de los grupos, así como los objetivos y normas de estos, moldean el uso que hacen de las noticias, y destacan el lugar entre las principales motivaciones para vincularse a estos grupos de WhatsApp y de Facebook. Sin embargo, no relacionan las características de las plataformas con las diferentes prácticas que se generan en los grupos investigados.

Este diferente significado asociado a las plataformas lo contempla Jennifer Alexander (2018) al describir el papel de los grupos digitales en la preservación de la identidad étnica de la tribu Levu Lahanan en Sarawak, tras la reubicación forzada por el gobierno de Malasia a fin de construir la presa de Bakun. La investigación, que combina técnicas clásicas con la observación etnográfica virtual, revela que estas comunidades han usado los grupos digitales en dos sentidos diferenciados: los grupos de WhatsApp permiten a los miembros de la diáspora enterarse de todo lo que ocurre en la tribu, y a través del grupo de Facebook regresan mediante su imaginación a su lugar natal, reforzando su arraigo al lugar. Como el autor advierte, la comunicación continua a través de 
Facebook ofrece más oportunidades para examinar los impactos sobre la identidad étnica y el vínculo con el lugar de origen.

\section{Consideraciones finales}

La aún incipiente bibliografía producida en torno al fenómeno comunicativo de los grupos digitales muestra que los enfoques, las temáticas y los métodos y técnicas aplicadas presentan diferencias, según se aborden los grupos creados en la aplicación de mensajería WhatsApp o en la red social Facebook. Dicha disimilitud se asienta en la percepción de los propios investigadores de que ambas aplicaciones ofrecen diferentes oportunidades como campo de investigación.

WhatsApp es una herramienta de mensajería móvil y por ello tiene algunos rasgos distintivos como la velocidad en el intercambio, el bajo consumo, la volatilidad de los mensajes y la percepción de una cierta privacidad o intimidad. Esto hace de la aplicación un lugar donde se reproducen "las condiciones naturales de la conversación presencial" (BARGAS; MAIA, 2017, p. 40). De acuerdo con esta idea, las investigaciones realizadas hasta el momento apuntan que los grupos de WhatsApp son un campo propicio para observar de primera mano las interacciones entre los actores investigados y sus procesos de micro-coordinación, o para profundizar en el análisis conversacional y en la lingüística aplicada. Por ello, las investigaciones que estudian estos escenarios se centran en la red de vínculos que se teje a través de las interacciones cotidianas de los miembros, en las funciones de estos intercambios y en los usos lingüísticos, en especial los mecanismos que dan lugar a un tipo de 'escritura oralizada' (YUS, 2017).

Por el contrario, la red social Facebook se caracteriza por rasgos como la persistencia o almacenamiento, la opción de realizar búsquedas y recuperar las publicaciones en el futuro, la edición y replicabilidad de los contenidos y la existencia de una audiencia invisible que, debido a la apertura de esta red, tiende a ampliarse más allá de los límites de los conocidos, especialmente en el caso de los grupos (BOYD, 2007). Si a estas cualidades sumamos la disposición cronológica de las publicaciones en 
forma de línea de tiempo (UREVICH, 2016), podemos entender que Facebook sea comprendida como una red social adecuada para producir un relato, fundamental en los procesos de producción identitaria e ideológica. Por este motivo, en las investigaciones sobre los grupos de Facebook predominan los análisis del discurso orientados a conocer los elementos que intervienen en la construcción y representación de estas identificaciones.

Además, hemos podido advertir que en la aplicación WhatsApp suele estudiarse las interacciones de colectivos que comparten un entorno físico o marco contextual común, con el fin de coordinar sus acciones; esto tiene relación con el hecho de que conversar en WhatsApp suele implicar un alto nivel de compromiso (O'HARA et al., 2014). Sin embargo, Facebook aporta un lugar público y visible en el que pueden auto-representarse aquellos grupos sociales que reivindican un territorio propio, ya sea simbólico o físico, como los colectivos no heteronormativos, las mujeres, los jóvenes de las periferias, los migrados que desean conservar el vínculo con el origen, los que reivindican una autonomía territorial, o desean representar su propia visión del territorio frente al discurso único de los medios de masas, los que tratan de recrear esa topografía afectiva que ya solo existe en su memoria y en las fotografías o aquellos que promueven ideologías que no encuentran cabida en ambientes democráticos. 
Cuadro 1. Resumen enfoques, temas y métodos.

\begin{tabular}{|c|c|c|c|}
\hline & Enfoque & Temas & Métodos \\
\hline \multirow{4}{*}{ WhatsApp } & \multirow{2}{*}{$\begin{array}{l}\text { Significado de las } \\
\text { interacciones }\end{array}$} & Coordinación & \multirow{4}{*}{$\begin{array}{l}\text { Etnografía clásica: gru- } \\
\text { pos de discusión } \\
\text { Análisis conversacional } \\
\text { Lingüística aplicada }\end{array}$} \\
\hline & & $\begin{array}{l}\text { Efecto en los } \\
\text { vínculos sociales }\end{array}$ & \\
\hline & \multirow{2}{*}{ Usos del lenguaje } & $\begin{array}{l}\text { Funciones del } \\
\text { lenguaje }\end{array}$ & \\
\hline & & Escritura oralizada & \\
\hline \multirow{2}{*}{ Facebook } & $\begin{array}{l}\text { Producción } \\
\text { ideológica }\end{array}$ & $\begin{array}{l}\text { Minorías políticas } \\
\text { Ideologías } \\
\text { reaccionarias }\end{array}$ & \multirow{2}{*}{$\begin{array}{l}\text { Etnografía clásica: } \\
\text { entrevistas } \\
\text { Análisis de discurso }\end{array}$} \\
\hline & $\begin{array}{l}\text { Construcción } \\
\text { identitaria }\end{array}$ & $\begin{array}{l}\text { Nuevas culturas } \\
\text { Identidad } \\
\text { territorial }\end{array}$ & \\
\hline
\end{tabular}

Fuente: Elaboración propia.

Dadas estas diferencias en los enfoques, temáticas y en los abordajes metodológicos, animamos a desarrollar investigaciones comparadas que contribuyan a confirmar o refutar la conclusión a la que nos ha llevado esta revisión bibliográfica: que el carácter narrativo de Facebook es más propicio para construir historias o narraciones que nutren los procesos de producción de sentido, mientras que el lenguaje conversacional de WhatsApp tiene una alta dosis de oralidad, que también resulta fundamental para producir interacciones significativas, que generan un sentimiento de convivencia y estrechan los vínculos entre los miembros de una comunidad de prácticas.

\section{Referências}

ACOSTA, G. L. Gestión de pasiones y polarización en las redes sociales. Un análisis del aplicativo Grupos en Facebook. Discurso \& Sociedad, v. 6, n. 4, p. 684-719, 2012. AGUILAR-FORERO, N. Ciberactivismo y olas de agitación comunicativa. Consideraciones etnográficas. Íconos - Revista de Ciencias Sociales, n. 59, 2017.

AHAD, A. D.; LIM, S. M. A. Convenience or Nuisance?: The 'WhatsApp’ Dilemma. Procedia - Social and Behavioral Sciences, v. 155, p. 189-196, 2014. 
AKÇALI, E. Facebook: An Emerging Arena for Politics of Self-Determination in northern Cyprus? South European Society and Politics, v. 24, n. 4, p. 513-533, 2019.

ALEXANDER, J. Migration, Imagination and Identity in Modern Sarawak. Asia Pacific Journal of Anthropology, v. 19, n. 3, p. 264-280, 2018.

ALI, N. M. Sudanese Women's Groups on Facebook and \#Civil_Disobedience: Nairat or Thairat? (Radiant or Revolutionary?). African Studies Review, v. 62, n. 2, p. 103-126, jun. 2019.

ÁLVAREZ GANDOLFI, F. Problemáticas en torno de las ciberculturas. Una reflexión sobre las posibilidades y los límites de la etnografía virtual. Cultura, Lenguaje y Representación, v. 16, n. 2, p. 7-20, 2016a.

ÁLVAREZ GANDOLFI, F. Cibercultura otaku. Un análisis interdiscursivo de identidades fan puestas en escena en grupos de Facebook. Perspectivas de la Comunicación, v. 9, n. 2, p. 31-57, 2016b.

ARDÉVOL, E.; GÓMEZ-CRUZ, E. Digital Ethnography and Media Practices. In: DARLING-WOLF, Fabienne et. al. (Eds.). The International Encyclopedia of Media Studies. Oxford, UK: John Wiley \& Sons, Ltd, 2013. v. VII, p. 498-518.

ARZADON, M. M. C. E. Where machines rant, recite poems, and take outrageous selfies: An ethnography of a teachers' facebook group. Studies in Educational Ethnography, v. 13, p. 65-96, 2016.

BARBOSA, S.; MILAN, S. Do Not Harm in Private Chat Apps: Ethical Issues for Research on and with WhatsApp. Westminster Papers in Communication and Culture, v. 14, n. 1, p. 49-65, 2019.

BARGAS, J. DE K. R.; MAIA, R. C. M. Quilombolas no WhatsApp: o papel do aprendizado coletivo nas lutas por reconhecimento. Comunicação Mídia e Consumo, v. 14, n. 41, p. 31, 2017.

BOYD, D. Social Network Sites: Public, Private, or What? Social Network Sites Mediated Publics. Knowledge tree, p. 1-7, 2007.

CAPOGROSSI, M. L.; MAGALLANES, M. L.; SORAIRE, F. Los desafíos de Facebook. Apuntes para el abordaje de las redes sociales como fuente. Revista de Antropología experimental, n. 15, p. 47-63, 2015.

CHAGAS, V.; MODESTO, M.; MAGALHÃES, D. O Brasil vai virar Venezuela: medo, memes e enquadramentos emocionais no WhatsApp pró-Bolsonaro. Esferas, n. 14, p. 1, 2019.

CLIFFORD, S.; SMITH, J. A.; CHRISTIE, B. You See the Very Sharp Edge of the Problems of Prejudice in Town: Youth Service Providers' Perceptions of a Regional Community Facebook Group. Social Media and Society, v. 6, n. 1, 2020.

DA SILVA BRAGA, R. Technologies as a means, meetings as an end: Urban interactions of a migrant community in rio de Janeiro, Brazil, mobilized through WhatsApp. IFIP Advances in Information and Communication Technology, v. 490, p. 68-81, 2016.

DAVIS, J. L.; CHOUINARD, J. B. Theorizing Affordances: From Request to Refuse. Bulletin of Science, Technology ש Society, v. 36, n. 4, p. 241-248, 2016. 
DE SOUSA, M. T. Rezando pelo smartphone: reconfigurações de práticas religiosas em um grupo de oração católico pelo WhatsApp. Fronteiras - estudos midiáticos, v. 20, n. 2 , p. 217-230, 2018.

DÍAZ BIZKARGUENAGA, K. FaceGUK: la construcción social de la identidad vasca analizada desde la etnografía digital. Athenea digital, v. 15, n. 2, p. 275-288, 2015.

DONCEL, E. B. Circulación de memes en WhatsApp: ambivalencias del humor desde la perspectiva de género. Empiria, n. 35, p. 21-45, 2016.

DOS SANTOS, K. K. A.; SILVA, J. E. P. Que língua é essa? Estudo dos marcadores conversacionais do internetês no WhatsApp. Tomé: Universidade Federal do Pará, 2018. DURÁ-LIZÁN, M.; TRAVÉ-MOLERO, R.; NOGUÉS-PEDREGAL, A. M. Polimedia y grupos públicos: un estudio comparado sobre los procesos de selección de plataformas digitales. Miguel Hernández Communication Journal, v. 12, n. 1, p. 223-242, 2021.

EKMAN, M. Anti-immigration and racist discourse in social media. European Journal of Communication, v. 34, n. 6, p. 606-618, 2019.

ESTALELLA, A.; ARDÈVOL, E. Ética de campo: Hacia una ética situada para la investigación etnográfica de internet. Forum Qualitative Sozialforschung, v. 8, n. 3, 2007. EVANGELISTA, R.; BRUNO, F. WhatsApp and political instability in Brazil: targeted messages and political radicalisation. Internet Policy Review, v. 8, n. 4, p. 1-23, 2019.

FERRAUDI CURTO, M. C. Después de la protesta: Facebook y los lazos políticos locales. Avá, Revista de Antropología, v. 32, p. 131-152, 2018.

FUENTES GUTIÉRREZ, V.; GARCÍA DOMINGO, M.; ARANDA LÓPEZ, M. Grupos de clase; grupos de WhatsApp. Análisis de las dinámicas comunicativas entre estudiantes universitarios. Prisma Social, v. 18, p. 144-171, 2017.

GARCÍA HUERTAS, J. G.; GARRIDO PINTADO, P.; BOTAS LEAL, D. Interacción vecinal a través de Facebook. Análisis de un caso práctico. ACTAS ICONO14 - VI Congreso Internacional Ciudades Creativas. Anais, 2018.

GIUS, C. Online communities as points of observation of the transnational migrant career: a case study on Italian immigrants in Toronto. Journal of Ethnic and Migration Studies, v. 0, n. 0, p. 1-16, 2019.

GREGORY, J. Connecting with the past through social media: The Beautiful buildings and cool places Perth has lost Facebook group. International Journal of Heritage Studies, v. 21, n. 1, p. 22-45, 2015.

GUTIÉRREZ MARTÍNEZ, A. P. Etnografía móvil: una posibilidad metodológica para el análisis de las identidades de género en Facebook. Revista Interdisciplinaria de Estudios de Género, v. 2, n. 4, p. 26-45, 2016.

GÜZEL KORVER, S.; KESKIN, S. Sosyal Medyada Roman Etnisitesinin Öz-Temsili ve Ötekili in Sosyo-Dijital Telafisi. Connectist: Istanbul University Journal of Communication Sciences, p. 51-84, 2019.

HARLOW, S. Reconfiguring and remediating social media as alternative media: Exploring youth activists' digital media ecology in El Salvador. Palabra Clave, v. 19, n. 4, p. 997-1026, 2016. 
HINE, C. Ethnography for the Internet: Embedded, Embodied and Everyday. Huntingdon, GBR: Bloomsbury Publishing, 2015.

ITURRALDE CHAPARRO, E. Chairos contra Derechairos: El trolling y otras prácticas incendiarias de la comunicación en línea en un grupo temático sobre Historia, en Facebook. In: BARREDO, D.; CUNHA, M. R.; HIDALGO, J. (Eds.). Jóvenes, participación y medios de comunicación digitales en América Latina. La Laguna, Tenerife: Sociedad Latina de Comunicación Social, 2019. p. 103-132.

KÖNIG, K. Stance taking with 'laugh' particles and emojis - Sequential and functional patterns of 'laughter' in a corpus of German WhatsApp chats. Journal of Pragmatics, v. 142, p. 156-170, mar. 2019.

KURNIASIH, N.; RIYADHSYAH, T. Virtual Ethnography Study of Inter-lecturer Communication in National Young Lecturers Forum WhatsApp Group. Proceedings of the 8th International Conference of Asian Association of Indigenous and Cultural Psychology (ICAAIP 2017). Anais...Paris, France: Atlantis Press, 2018

LING, R.; LAI, C.-H. Microcoordination 2.0: Social Coordination in the Age of Smartphones and Messaging Apps. Journal of Communication, v. 66, n. 5, p. 834-856, 2016.

MARICHAL, J. Political Facebook groups: Micro-activism and the digital front stage. First Monday, v. 18, n. 12, 2013.

MARRERO SANTANA, L.; JIMÉNEZ HERNÁNDEZ, A.; RODRÍGUEZ BRITO, A. Usos sociales de Facebook que realizan jóvenes cubanos residentes en el país. Index. comunicación: Revista científica en el ámbito de la Comunicación Aplicada, v. 7, n. 3, p. 187-209, 2017.

MATHUR, N. A Petition to Kill: Efficacious arzees against big cats in India. Modern Asian Studies, v. 53, n. 1, p. 278-311, 2019.

MCGREGOR, M. et al. Talking about chat at work in the global south: An ethnographic study of chat use in India and Kenya. Conference on Human Factors in Computing Systems - Proceedings, 2019.

MENESES, D. Con Mis Hijos No Te Metas: un estudio de discurso y poder en un grupo de Facebook peruano opuesto a la «ideología de género». Anthropologica, v. 37, n. 42, p. 129-154, 2019.

MOLS, A.; PRIDMORE, J. When citizens are "actually doing police work": The blurring of boundaries in whatsapp neighbourhood crime prevention groups in the Netherlands. Surveillance and Society, v. 17, n. 3-4, p. 272-287, 2019.

MOSCONI, G. et al. From Facebook to the Neighbourhood: Infrastructuring of Hybrid Community Engagement. Computer Supported Cooperative Work: CSCW: An International Journal, v. 26, n. 4-6, p. 959-1003, 2017.

NAZARUDDIN, M. Social media and alternative discourse on natural hazard: A case study of facebook group 'info merapi'. Jurnal Komunikasi: Malaysian Journal of Communication, v. 36, n. 1, p. 480-494, 2020.

O'HARA, K. P. et al. Everyday dwelling with WhatsApp. Proceedings of the 17th ACM conference on Computer supported cooperative work \& social computing - CSCW'14. Anais...New York, New York, USA: ACM Press, 2014 
436 IDENTIDAD TERRITORIAL

PADILLA HERRADA, M. S. Formas de expresión de la modalidad confirmativa en WhatsApp. Pragmalinguistica, n. 25, p. 439-466, 2017.

PÉREZ-SABATER, C. Discovering language variation in WhatsApp text interactions. Onomazein, v. 31, n. 1, p. 113-126, 2015.

PROCTOR, D. Policing the Fluff: The Social Construction of Scientistic Selves in Otherkin Facebook Groups. Engaging Science, Technology, and Society, v. 4, p. 485, 2018.

RAMÍREZ PLASCENCIA, D. "I make Mexico at my home": social media usage, diasporic discourse, and cultural endurance of Mexicans living in Barcelona, Spain. OBETS. Revista de Ciencias Sociales, v. 15, n. 1, p. 233, 29 jun. 2020.

RESENDE, G. et al. A System for Monitoring Public Political Groups in WhatsApp. Anais do Simpósio Brasileiro de Sistemas Multimídia e Web (WEBMEDIA). Anais... Sociedade Brasileira de Computação - SBC, 16 oct. 2018

REVILLA, B. P. Shaping energy norms in digital communities: The contribution of online discussion boards to questioning energy needs in Amsterdam. Energy Research 6 Social Science, v. 67, 2020.

RIQUELME, C. M.; SOLÍS, R. G.; GALLEGOS, O. B. Online sociability in youth groups of facebook in Chile. A study through virtual ethnography. Obets, v. 13, n. 1, p. 151-173, 2018.

SÁDABA, I.; BARRANQUERO, A. The social networks of cyberfeminism in Spain: Identity and action repertoires. Athenea Digital, v. 19, n. 1, p. 1-24, 2019.

SWART, J.; PETERS, C.; BROERSMA, M. Sharing and Discussing News in Private Social Media Groups. Digital Journalism, v. 7, n. 2, p. 187-205, 2019.

TABARES HIGUITA, L. X. Análisis del discurso violento y de odio en dos grupos de Facebook contra la candidatura de Rodrigo Londoño 'Timochenko' a la presidencia de Colombia. Index Comunicación, v. 8, n. 3, p. 157-183, 2018.

TORRES, P. Seguridad y separación clasista en Córdoba: grupos de Whatsapp y aplicaciones de asistencia ciudadana para la gestión de la conflictividad. Astrolabio Nueva Época, v. 18, p. 9-33, 2017.

TUR-VIÑES, V.; FERRÁNDIZ, R. R. Transmedialidad: series de ficción y redes sociales. El caso de Pulseras Rojas en el grupo oficial de Facebook (Antena 3. España). Cuadernos.info, v. 34, n. 1, p. 115-131, 2014.

UREVICH, A. El tiempo todo en Facebook. Aposta, v. 69, p. 217-238, 2016.

VELA DELFA, C.; CANTAMUTTO, L. De participante a observador: el método etnográfico en el análisis de las interacciones digitales de Whatsapp. Tonos Digital, Revista Electrónica de Estudios Filológicos, v. 31, 2016.

VERMEER, S. A. M. et al. WhatsApp with Politics?!: Examining the Effects of Interpersonal Political Discussion in Instant Messaging Apps. The International Journal of Press/ Politics, v. 0, n. 0, p. 1-28, 17 jun. 2020.

YAZAN, B. Adhering to the language roots: Ottoman Turkish campaigns on Facebook. Language Policy, v. 14, n. 4, p. 335-355, 2015. 
YUS, F. Los efectos de internet y las redes sociales en el español. The British Journal of Psychiatry, v. 111, n. 479, p. 1009-1010, 2017.

\section{Sobre os autores}

Mercedes Durá-Lizán - Personal investigador Departamento de Ciencias Sociales y Humanas. El autor realizó la revisión bibliográfica, el análisis y categorización de los textos y el borrador inicial del artículo.

Antonio Miguel Nogués Pedregal - Profesor titular del Departamento de Ciencias Sociales y Humanas e investigador principal del grupo de investigación Culturdes de la Universitas Miguel Hernández. Es visiting scholar en las Universidades de Oxford, Ljubljana y Johannes Gutenberg en Mainz. Los resultados de investigación han sido publicados en revistas nacionales e internacionales y traducidos al inglés, italiano y alemán. Ha coordinado la edición de Cultura y turismo and Culture and Society in Tourism Contexts. Tiene tres tramos de investigación reconocidos por ANECA y cuatro quinquenios docentes. Es coordinador de varios proyectos transnacionales en programas de la Unión Europea. El autor ha participado en la concepción de la investigación y en la revisión del manuscrito.

Data de submissão: 03/08/2020

Data de aceite: 17/11/2020 